\title{
Correction to: Speech Technology
}

Fang Chen and Kristiina Jokinen

Correction to:

F. Chen, K. Jokinen (eds.), Speech Technology, https://doi.org/10.1007/978-0-387-73819-2

The book was inadvertently published with wrong Book Editor name. The correct Editor name is Kristiina Jokinen rather than Huggins. 